Relations industrielles

Industrial Relations

\title{
The Selection of Trainees Under MDTA, Institute of Management and Labor Relations, Rutgers - The State University, New Brunswick, New Jersey, 124 pages.
}

\section{Paul Bouchard}

Volume 22, numéro 2, 1967

URI : https://id.erudit.org/iderudit/027804ar

DOI : https://doi.org/10.7202/027804ar

Aller au sommaire du numéro

Éditeur(s)

Département des relations industrielles de l'Université Laval

ISSN

0034-379X (imprimé)

1703-8138 (numérique)

Découvrir la revue

Citer ce compte rendu

Bouchard, P. (1967). Compte rendu de [The Selection of Trainees Under MDTA, Institute of Management and Labor Relations, Rutgers - The State University, New Brunswick, New Jersey, 124 pages.] Relations industrielles / Industrial Relations, 22(2), 309-309. https://doi.org/10.7202/027804ar

Tous droits réservés @ Département des relations industrielles de l'Université Laval, 1967
Ce document est protégé par la loi sur le droit d'auteur. L’utilisation des services d'Érudit (y compris la reproduction) est assujettie à sa politique d'utilisation que vous pouvez consulter en ligne.

https://apropos.erudit.org/fr/usagers/politique-dutilisation/ 
des résultats clés qui serviront par la suite à mesurer ses progrès. D'où l'établissement de différents programmes ayant à peu près les mêmes buts, soit le meilleur service possible qui opportera le meilleur profit possible tout en coordonnant les efforts des employés vers le même but, c'est-à-dire le succès de l'entreprise.

Dans la quatrième partie, l'auteur traite des différents programmes qui ont été tracés par environ 23 compagnies afin que les buts généraux de chacune de ces compognies soient en accord avec les buts de chaque employé. On y examine principalement les difficultés rencontrées, le manque de participation et de motivation de la part des employés, la manière avec laquelle les compagnies ont établi des standards ainsi que le nombre d'objectifs qui doivent être etublis pour un trovail donné.

L'outeur, dans la cinquième partie, revient un peu sur ce qui a été dit dans le chapitre 2, mais il fait ceci dans le dessein d'approfondir davantage l'importance qu'il $y$ a de ne pas séparer les objectifs et les standards de performance en ce qui a trait ò certaines activités qui sont d'une importance primordiale pour la direction, telles que le budget, les plans d'organisation et les descriptions de tâches, ainsi que l'évoluation de la performance et le développement de la main-d'oeuvre.

Pour conclure, l'auteur nous parle des aspects du futur en ce qui regarde l'entreprise. II voit celle-ci comme un système dynamique dans lequel les décisions programmiées et l'emploi des standards de performance se multiplieront et dans lequel le processus d'information sera de beaucoup amélioré

\section{Paul BELANGER}

\section{The Selection of Trainees Under MDTA,}

Institute of Management and Labor Relations, Rutgers - The State University, New Brunswick, New Jersey, 124 pages.

Cet ouvrage a pour objet l'examen du processus de sélection pour les travailleurs susceptibles de suivre des cours de formation professionnelle et la délimitation des caractéristiques des personnes choisies ou recalées et de celles qui n'ont jamais fait application pour suivre de tels cours.

Le présent rapport est le premier d'une série de deux. Le second permettro le développement de techniques spéciales de forma- tion professionelle pour les personnes qui ne sont pas capables de rencontrer les exigences sélectives, c'est-à-dire les personnes qui combinent une formation éducative inadéquate, de faibles aptitudes et une basse motivation.

Quant au contenu proprement dit du projet lui-même, disons qu'il se divise en trois sections. Dans une première section, les sujets qui y sont étudiés sont successivement la position du problème lui-même, le contexte où se situe l'étude, la population qui est l'objet de l'analyse, la classification de cette même population en fonction des règlements du MDTA, les échantillons choisis et finalement le plan du rapport.

La deuxième section, de son côté, concerne les caractéristiques des candidats qui se présentent aux progrommes du MDTA. On retrouve, par conséquent, des caractéristiques démographiques, des caractéristiques psychologiques et enfin des caractéristiques relatives au stotut occupationnel des travailleurs.

Pour terminer, le rapport retrace l'expérience préalable des travailleurs et élabore également sur leur expérience postérieure à leur période de formation professionnelle. II ne faudrait pas minimiser non plus l'importance que nous devons accorder à la description quelque peu sommaire du nombre et des divers types de cours organisés à partir des mesures gouvernementales du MDTA.

Si nous avions une appréciation à donner sur ce rapport, nous dirions qu'il est en mesure d'intéresser au plus haut point les responsables de la préparation et de la structuration des cours de formation professionnelle pour les travailleurs. Le problème de la sélection des candidats aux cours de formation professionnelle n'est pas encore résolu. Nous en sommes à la période d'essais et de tentatives. D'une part, il y a l'impératif d'un résultat positif toujours plus grand à atteindre et d'autre part, il y a le nombre passablement imposant de personnes rejetées en raison souvent de critères plutôt discutables. En fait, c'est le moment d'une prise de conscience et d'line remise en question des critères actuels qui devraient conduire à plus ou moins longue échéance à la reformulation ou au remplacement des critères actuels.

\section{Paul BOUCHARD}

L'importance croissante du secteur des services dans les pays membres de I'OCDE, por Maurice Lengellé, Organisation de Coopération et de Développement Economique, Paris, 1966, 144 pages. 